\title{
Synchronicity, Mind, and Matter
}

\author{
Wtodzistaw Duch \\ Nicholas Copernicus University \\ Torun, Poland
}

\begin{abstract}
Experiments with remote perception and Random Event Generators (REG) performed over the last decades show small but significant anomalous effects. Since these effects seem to be independent of spatial and temporal distance, they appear to be in disagreement with the standard scientific worldview. A very simple explanation of quantum mechanics is presented, rejecting all unjustified claims about the world. A view of mind in agreement with cognitive neuroscience is introduced. It is argued that mind and consciousness are emergent properties of the brain and are understandable without any nonphysical assumptions. A plausible explanation of the results of anomalous experiments, based on the concept of synchronicity, introduced by C.G. J ung and advocated by W. Pauli, is offered. A proof is given that strong correlations should exist between any systems that once interacted. Synchronicity events between parts of the brain and physical objects may be sufficient to explain the results of anomal ous experiments. Standard physics is sufficient to understand these phenomena.
\end{abstract}

Motto:

It would be most satisfactory if physics and psyche could be seen as complementary aspects of the same reality.

—W. Pauli (In J ung \& Pauli, 1952/1973)

\section{Introduction: On Mind and Matter}

$\mathrm{P}$ UBLICATION of the Pauli-J ung correspondence (Pauli \& J ung, 1992) leaves no doubt that Wolfgang Pauli devoted much thought to the concept of synchronicity, or the acausal synchronicity of meaningful events. This concept was introduced by C. G. J ung (J ung \& Pauli, 1952/1973) in a book that al so includes Pauli's contribution. Despite many discussions of synchronicity that Pauli had with scientists working at the Institute for Advanced Study in Princeton, USA (where he spent his war years), the idea was somehow abandoned. Physicists were not ready to discuss acausal coinci dences between events distant in time and space, mental experiences (dreams, intentions, thoughts), and meaning. Pauli himself was famous for creating trouble in laboratories he visited, and apparently he regarded this "Pauli effect" as a manifestation of synchronicity. The wish that Pauli expressed more than forty years ago to see physics and psycheas complementary aspects of the same reality may slowly become manifest now, thanks to our deeper understanding of the foundations of physics and the development of the cognitive sciences.

Perceptual and cognitive processes are not passive but involve fitting the best models to the incoming data. Perceiving three-dimensional objects with colors that are almost independent of illumination requires many assumptions that the brain has learned to make in the course of evolution. Active perception leads to the metaphor of the brain as the "machine generating meaning" (Freeman, 1996), discriminating and evaluating everything from a subjective perspective. It is sufficient to see only those aspects of reality that may influence our decisions, so we do not see more. Looking for meaning is a great strategy facilitating survival in typical situations, but it also leads to finding meaningful patterns in random dots or shapes of the clouds. At the cognitive level the situation is analogous. We assume that "we" know "ourselves," but how can we really know? We know by observing and making theories about our own behavior (Gopnik, 1993). 
These theories lead to deeply ingrained illusions about our minds.

Understanding means relating new facts to old theories and personal beliefs. Cognitive science is based on systematic observations, theories that cannot be easily replaced by alternative ones. So many details about cognition are now understood that the introduction of new paradigms may be very difficult. The difficulties of a mathematical description of nature are progressively increasing at each step: classical physics is able to solve the two body problem exactly; in relativistic quantum mechanics exact solutions are obtained only for the one particle case; in quantum electrodynamics only the vacuum problem (zero particles) is exactly solvable; while in quantum chromodynamics even the vacuum problem is too difficult to be exactly solvable. F uturegrand unified theories will not be easier to understand or to apply. The solution of the conceptual problems of physics and the cognitive sciences and the description of physical phenomena involving ordinary matter cannot lie in exotic physical theories. A unified view of mind and matter should be possible al ready within the present paradigms, or we may not be able to understand such a theory at all.

Since paradigms are so hard to change there is a natural tendency to dismiss all evidence that does not fit into an existing framework. In the last decades, a large number of experiments involving human operators have been performed, giving data that seem to be hard to understand within the scientific framework ( ahn \& Dunne, 1987; J ahn, 1982; Schmidt, 1993; Puthoff \& Targ, 1976). The effects observed were analyzed in traditional terms derived from parapsychology, such as psychokinesis, tel epathy or precognition. More recent terms include "anomalous data," "remote viewing," "remote perception," or "human operator effects." These results are largely ignored as impossible, or threatening to the scientific worldview. Hope that a simple systematic error explaining such data will be discovered does not seem to be justified. Data pointing to the existence of such effects are accumulating. In particular, solid evidence for what is called "remote perception" and for the influence of intentional mind states on random events, including past and future events, has been accumulated.

The last type of effect has also been called a "micropsychokinetic effect." A large amount of experimental data has been obtained using the Random Event Generators (REGs) in the
Princeton Engineering Anomalies Research (PEAR) laboratory, USA (J ahn \& Dunne, 1987). The portable equipment and the software for performing such experiments is now available from this laboratory to other scientists interested in experimenting on their own. These effects seem to represent the reproducible synchronicity events that Pauli called for in his letter to Fierz (quoted in Laurikainen, 1988): “F or me personally it would be much nicer to begin with 'acausal orderings' which are al ways reproducible (including those of quantum physics) and attempt to understand the psycho-physical connections as a special case of this general species." Perhaps the time is ripe for such an attempt.

The explanation of such experiments is very difficult for two reasons: the involvement of human operators, and our persistent illusions about reality. Quantum mechanics is presented first as a remedy for such illusions. Mind as an emergent property of the brain is presented next. Physical and mental points of view are combined to give a plausible explanation of the observed data as a special case of synchronicity. In contrast to many other explanations of anomal ous phenomena, no extensions to physics used in the description of matter, nor cognitive science used in the description of minds, is needed. The result is a reasonable, unified view of mind and matter.

\section{Quantum Correlations}

$\prod \begin{aligned} & \text { he progress of science is the history of shed- } \\ & \text { ding illusions and false assumptions. At the }\end{aligned}$ beginning of the twenti eth century scientists assumed that nature works according to the principles of classical mechanics. I deas about reality were mistaken for reality itself. Positions, forces, inertia or momenta, have well-grounded intuitive meanings referring to feedback from body movements. Any object-human, tree, or atomis reduced to a rather small set of properties that human minds are able to conceive. Instead of a full, infinitely subtle description of an object ("infinity in a grain of sand," as Blake puts it), many ideas about space, time and movement are projected onto reality. In fact, each object is infinitely complex, and thus it should be represented by a vector $\mid \mathrm{O}(\mathrm{t})>$ with an infinite number of components, describing its properties (its state) at timet.

The state vector should contain perfect knowledge about the object, allowing for determination 
of any property P. A measurement is needed to determine it, requiring an interaction with the object O. F or example, in order to look at a glass, scattered light is needed to see it, and eyes and brain (or camera and computer) are needed to estimate its position, shape, size, and color. The measuring procedure is symbolized by a certain operator $\mathbf{P}$ acting on the vector, $\mathbf{P}|\mathrm{O}\rangle$. In return, a value of the measured property $\lambda_{p}$ is obtained, and the state of the object $|\mathrm{O}\rangle$ remains unchanged. This measurement procedure is summarized in symbolic form as:

$$
\mathbf{P}|\mathrm{O}\rangle=\lambda_{\mathrm{p}}|\mathrm{O}\rangle
$$

This is the basic equation of quantum mechanics, called eigenequation for the operator $\mathbf{P}$ corresponding to some observable property. No assumptions about the world are required to apply this general procedure. If $|\mathrm{O}\rangle$ represents a human and $\mathbf{P}$ a procedure of asking questions then $\lambda_{p}$ is a verbal answer. To measure classical physics properties, such as energy, position, momentum or timeflow, an appropriate operator is needed. In psychol ogy and physics the answers to two questions $\mathbf{P}, \mathbf{Q}$ that cannot be answered at the same time may depend on the order of the questions. This is the cel ebrated Heisenberg relation, $\mathbf{P Q}|\mathrm{O}\rangle \neq \mathbf{Q P}|\mathrm{O}\rangle$. For example, the position and momentum of a particle cannot be measured at the same time. Localization of a particle increases the uncertainty of its momentum.

All matter shows wave-like properties if an operation $\mathbf{P}$ to measure them is set up. There fore, a good mathematical representation of a state vector for an elementary particle is a wave function $|\Psi\rangle$. Setting up the mathematical structure of such a theory may be done in many ways and is a technical matter. The momentum operator $\mathbf{p}$ is proportional to the changes of the $|\Psi\rangle$ wave between two points (i.e., to the derivative of $|\Psi\rangle$ in respect to position), while the kinetic energy operator $\mathbf{H}$ (by analogy to classical mechanics called the Hamilton operator) is proportional to the square of the momentum $\mathbf{p}^{2}$. This operator represents a measurement of energy of a system described by a state vector $|\Psi\rangle$ :

$$
\mathbf{H}|\Psi\rangle=\mathrm{E}|\Psi\rangle
$$

This is the famous Schrödinger equation. It is a symbolic representation of the general principle: to know some property prepare a measurement procedure $\mathbf{H}$ acting on the object in a given state $|\mathrm{O}\rangle=|\Psi\rangle$.
Quantum mechanics allows only for predictions of results of the measurements. What has not been measured should not be claimed. The answers depend on the questions that are posed. Nature shows different faces in different experiments. This is not a unique property of microobjects. Many properties of people also depend on the questions that are asked and the details of experimental arrangements. As long as objects are not changed by interactions in the measurement process, the same values of their properties are obtained in repeated experiments. This is usually the case with macroscopic physical properties. In the real $m$ of complex systems with internal structure, such as minds, and in the realm of very small objects, such as atoms, interactions always change state vectors.

Elementary objects are absolutely identical. Two objects that once were part of a quantum system (i.e., interacted with each other) should be described by a common state vector. Even when these objects become separated at a large distance they still form one system. Only by measuring their properties, and finding no correlations between the results, may the independence of the objects be established. There are two kinds of correlations. Trivial correlations result from conservation laws: If the total object had zero momentum, and after separation one part had momentum p, then the other part should have momentum - p. Finding such correlations shows that the systems have interacted in the past. Nontrivial correlations concern properties that cannot be simultaneously measured.

In the hypothetical case of two people with identical views, it may still happen that some views change, depending on the order in which questions areasked. For example, question $\mathbf{P}$ may be about a favoritemusical instrument and question $\mathbf{Q}$ about a favorite musician who is playing it. Asking the first question prepares (primes) the subject for the second. If one person is asked the same questions, in the same order as the other person, the answer should be identical. However, if the order in which questions are asked does matter, $\mathbf{P Q}|\mathrm{O}\rangle \neq \mathbf{Q} \mathbf{P}|\mathrm{O}\rangle$, and questions are presented in random order, then two identical sets of answers are a sign of nontrivial correlations. Suppose the first person answers "trumpet" to question $\mathbf{P}$ and "Miles Davis" to $\mathbf{Q}$. The second person, asked about the musicians, first has several choices, but still answers "Miles Davis" and "trumpet." Although their views are identi- 
cal, the choices could have been different. If they are not, they are correlated in a nontrivial way.

Although no one has done such an experiment with people (learning a list of paired associations could be one possibility) we assumethat only trivial correlations between people are possible. Quantum mechanics predicts that tests with elementary objects, such as photons, electrons or atoms, should show nontrivial correlations. In fact, this was Einstein's main objection to quantum mechanics. His formulation of an apparent paradox (Einstein, Podolsky, \& Rosen 1935), known as the Einstein-Podolsky-Rosen (EPR) paradox, brought the deep problem of a description of separated systems in quantum mechanics to the attention of physicists. Systems that are described by a common state vector are called "entangled." The problem is, how can the state vector representing the whol e group of objects be broken into separate vectors that describetwo or more independent parts, that is, how to disentangle the system?

To summarize the main point of this section: From a theoretical point of view disentanglement is impossible. As Wolfgang Pauli once remarked: "Was Gott vereint hat, soll der Mensch nicht trennen" ("What God has united men should not separate"). One cannot start with the two independent vectors describing the system without "playing God's role." This became evident in the last two decades when very precise experiments measuring correlations between properties of pairs of particles separated at large distances were performed (Selleri, 1987). These correlations were in full agreement with the predictions of quantum mechanics, but could not be explained assuming that state vectors of particles were independent. Existence of these nontrivial correlations, called the EPR correlations, or correlations violating Bell's inequality (an inequality setting a limit on the magnitude of correlations between measurements for independent particles) was predicted by quantum mechanics. The experimental verification of these predictions (cf. Selleri, 1987) was a great success. Interactions with other particles create complex networks of nontrivially correlated events, making it difficult to measure correlations between pairs of particles. Independence is one of these illusions acquired in early childhood. It is an illusion in the same sense as the independent existence of space and time is an illusion, although in both cases Newtonian concepts are useful approximations. The heated debates about the meaning of these results showed how hard it is to give up such deeply held convictions.

Quantum mechanics (QM) is unable to describe the process of separation in which particles become independent (the proof is in the Appendix). Some experts came up with alternative theories announcing the "death of quantum mechanics" (Piron, 1985, p. 207) and presenting a theory of the quantum-logic type that allows for the existence of independent objects (Aerts, 1982). Despite all the successes of quantum mechanics, scientists keep projecting their own ideas onto reality, trying to tell nature how to behave. The ories that allow for separation have never been successful in predicting anything. How can one tell that the separated subsystems are really independent? Only by rejecting the temptation to make unjustifiable claims, and testing for correlations in carefully designed experiments. Correlations between several particles that have not been directly entangled are also measurable (Nielsen \& Chuang, 2000).

It is not clear how to apply a full quantum mechanical treatment to small systems, such as a crystal or a biomol ecule, in the neighborhood of a large system (Primas, 1981). Microscopic bodies cannot be isolated in a quantum mechanical sense, since they are al ways strongly coupled with their environment and thus should show nontrivial correlations. Different patterns of neural excitation may be modeled in quantum mechanical fashion as the eigenstates of some operators. Since two different patterns cannot exist at the same time, these operators cannot commute. An analog of Bell's theorem for such a model should establ ish a limit for correlations between two neural systems. Straightforward estimation of the correlation coefficient obtained in theAppendix shows that correlations should al ways belarge. Why is it, then, so hard to measure such effects? I nteraction with warm, macroscopic bodies (thermal degrees of freedom) may wash them out completely. Detailed investigation of this point brought Khalfin and Tsirelson (1992) to thecondusion: "Under very careful, but undoubtedly feasibleisolation of the collective degrees of freedom from the thermal ones, quantum correlations can arise and be conserved for long periods of time, even in the mechanical motion of macroscopic bodies." Such quantum mechanical correlations between two separated crystals should be induced by mechanical movement and should persist for a long time.
A.I.:

Provide

p.\#at

the end of quote. 
If the measurement on the first particle is performed after one second, and on the other particle one year after the separation, statistical correlations between the results may still be stronger than could be expected if the two particles were independent of each other. From another point of view, such correlations may look like an influence of the present measurements on the future or past measurements, or like precognitive or retrocognitive results. Quantum mechanics does not admit such interpretations, since this would be claiming more than the experimental results justify. Nontrivial correlations are acausal. Experimental devices are designed to measure only simple correlations, for example by counting photons. I nteractions among a large number of particles may be too subtle to be measured with equipment that is not sensitive to subtle changes in the correlated patterns. Is it possible that our brains are sensitive to such patterns? Are the remote viewing and the REG experiments simply another expression of this basic interdependence of nature?

\section{Brains and Minds}

$I^{N}$ N THE remote viewing and REG experiments, intentional states of mind are crucial. Consciousness is regarded by some physicists as an ill-defined force pervading the universe, something necessary to "collapse wave functions" and interfere directly in the measurement process. This idea has been proposed by $\mathrm{E}$. Wigner (Wigner, 1962, pp. 284-302), who gave it up later (Mehra \& Wightman, 1995, p. 271), when he understood the difficulties of maintaining coherent quantum states in the brain. Discussions of this topic still go on without any reference to real cognitive phenomena (Stapp, 1993; Penrose, 1994). Before the measurement thestate vector $|\mathrm{O}\rangle$ contains all possible outcomes of experiments. After the measurement they suddenly collapse to the observed value (e.g., the particle found at some position). The state vector is not a physical object but a collection of properties that are determined through measurements. The interaction of a quantum system with classical measuring apparatus has been successfully described without the need for conscious intervention (Giulini, J oos, Kiefer, Kupsch, Stamatescu, \& Zeh, 1996). Neurons are sufficiently large to be accurately described as classical systems. Quantum effects in neurons may be observed only at timescales shorter than one picosecond (Tegmark, 2000), therefore they have no influence on their normal functioning, which is a billion times slower.

Quantum and spiritual explanations postulate some mysterious processes that give rise to mental states, without really explaining anything. Why does a specific kind of damage to the brain dramatically change the inner world of the person? The only fruitful approach to such questions so far has been based on a natural assumption that minds are emergent properties of very complex brains. Mind is a complex of many faculties related to perception, cognition, emotion, thinking, planning, imagining, acting, maintaining a subjective view of the world and the self in it (Freeman, 1996). The inner world seems to have nothing in common with the brain and its state, being qualitatively quite different. How then can mind arise from the brain? Philosopher $\mathrm{H}$. Putnam calls it a "disastrous picture of the world," an explanation that is "more obscure than the phenomenon to be explained" (Putnam, 1978).

There are so many misconceptions here that this issue requires careful investigation. Brain is the substrate in which mental processes take place. Connections between information processing by different areas of the cortex and subcortical nuclei on the one hand, and mental functions and dysfunctions on the other, are well established (Ruppin, 1995). Although the brain is the most complex object in the known Universe, and experiments with human brains are technically very difficult, the neural sciences have recently made unprecedented progress, describing processes at levels ranging from the molecular to that of global brain dynamics (Gazzaniga, 1999). Mental functions result from information processing by highly specialized brain areas, and can be observed using brain imaging techniques. Electric and magnetic fields showing this activity may be correlated directly with mental experiences in monkeys (Leopold \& Logothetis, 1999), and the introduction of noninvasive techniques that will do the same with humans is just a matter of time. Consciousness will lose its mystery once we are able to observe, using brain imaging techniques, what goes on in the mind of the owner of the brain.

How can the inner world be a product of the brain? And how is it that sound is converted into el ectrical signals, compressed into a wire and sent over large distances? How is it possible that moving images, spatio-temporal structures, arestored in the form of binary patterns on a DVD disk? To
A.I.:

Provide p.\#at the end of quote. 
philosophers of a pretechnological era all these questions would have been equally puzzling. $\mathrm{Bi}-$ nary patterns on the disk areturned, with thehelp of appropriate hardware, into electrical signals that become bright and dark spots on a screen. Binary patterns encode the structure of the images and the electronic player with the TV set recreates the state of the camera's photosensitive element. The representation does not have to resemble the original-brain and mind states may be quite different. Similarity should be sought at another level. Recognizing danger starts a series of brain states, a part of a network with complex inner relations. A network of mind states exhibits similar relations. Second-order similarity, that is similarity of relations between states, rather than the states themselves, is sufficient to link mind with brain (Edelman, 1998).

Does this mean that mind may be reduced to the brain? Not at all. The brain is a product of millions of years of adaptations that enabled survival in a hostile, changing environment. Cognitive faculties have been framed by the needs of organisms. We pay attention to and notice only those events that have potential value or meaning for us. For example, col or constancy is achieved in a wide variety of illuminations, making it easy to discriminate between red and green fruits. Light reflected from surfaces carries much information that the brain removes to simplify the object recognition task (Shepard, 1993). The sensory cortex prepares the incoming signals to facilitate decisions taken by the mind at the highest level of control. At the mind level individual history, a subjective view of the world and acquired cognitive skills are responsible for taking actions, deriving meaning from the input signals.

Brain structures have evolved to support the mind, they exist only because they are useful to the mind. Understanding of the mind requires understanding of the subjective world, and of relati ons between different states of mind. Hearing the same mel ody brings very different associations to different minds. Understanding these associations requires understanding of local culture and individual history. Brain science stops at explaining general cognitive and affective mechanisms. Mind uses these mechanisms to create an inner, subjective world at an emergent, autonomous level. Animal behavior does not follow from anatomy and physiology, but requires understanding of evolution, environment, and social patterns of behavior. Biology is not reducible to chemistry. Neuro- physiological processes are needed to support the mind but do not explain it fully. If we knew all about the brain, westill would not understand the inner world of the individual. Mind states cannot be reduced to brain states.

Mind is based on states that the brain may potentially enter, relations between those states and operations transforming one state into another. Nothing el se is needed to explain the structure of experience. The sensorimotoric actions of primitive animals become inner actions, that do not always end in motor behavior, but transform one mind state into another. Seeing is a way of exploring the environment (O’Regan \& Noë, 2001). Brain states differ in a qualitative way: Seeing and hearing engage different areas of the brain, and seeing red and green colors are different states of the visual cortex. These brain states are associated with other states, creating for examplea statethat the mind evaluates as "a pleasant red evening sky" experience. The mind-body problem, which has been called the most serious obstacle to the advancement of science (Rakover, 1993), does not exist. The qualitative character of experience, called the "hard problem" of consciousness (Chalmers, 1996), is an obvious consequence of brain-mind relations. Any system (call it an "artilect") that works on brain-like principles, creating internal physical states sufficiently rich to respond to changing sensory stimulation, and being able to evaluate these states by making associations with memorized states, will claim to have experiences of different qualities. The comments of such an artilect will resemblethehuman stream of consciousness. For technical reasons, it is still very difficult to create human-like responses to sensory stimulations, and thus to createa detailed structure of the human-like mind.

Mathematical modeling is more powerful than merelinguistic description. Verbal descriptions are imprecise models of reality, while mathematical models may have an arbitrary degree of precision. Everything that can be expressed in words can also be subject to mathematical modeling. Things that cannot be expressed in words, continuous changes, can be treated using the formalism of dynamical systems. Cognitive science, aiming at understanding how the mind works, should be based on mathematical language. The lack of a proper languageto describe mind events is responsible for fundamental problems in the cognitive sciences. A Platonic model of the mind sketched in Duch (1994-1997) provides such a language. In 
some respects it is similar to Nalimov's probabilisticsemantics and program of geometrization in linguistics and psychology (Nalimov, 1985). Nalimov starts from logic and linguistics, defining the semantic field and probability distributions over this field. This brings him towards fuzzy theories of meaning, devel oped by L. Zadeh (Wang, 2001). The idea of a living world as a text, and semantics as something given, is a serious restriction on the applicability of Nalimov's approach. The Platonic model starts from brain dynamics, defining the space in which mental events take place.

Space-timeis an arena in which physical events take place. Cognition is dominated by vision, and geometrical concepts of physics are therefore useful metaphors. Kurt Lewin in 1938 proposed a similar language for psychology (Lewin, 1938). Mental events were taking place in a "psychological space" under the influence of "cognitive forces." George Kelly, in his psychology of personal constructs, also favored geometry instead of logics (Kelly, 1955). Some psychologists would like to use his ideas for a central theory in cognitive science (Shaw \& Gaines, 1992). Roger Shepard (1987, 1994) has done much to analyze the geometry of inner space, finding invariant laws of stimulus generalization in spaces based on nonlinear input transformations (multidimensional scaling). The Platonic model foll ows this line of reasoning, trying to connect it with the brain's dynamics. The name has been derived from a famous allegory of Plato: Mind events of which we are conscious are only shadows of true reality, the neurodynamics of the brain.

The real objects of mind are not words or abstract symbols but rather "chunks of experience," involving sensorimotoric, bodily reactions. Mind objects are combinations of many features determined by the low-level processing of the brain circuits. In analogy to natural objects in quantum mechanics, objects of mind are nondecomposable and multidimensional, experienced in a unified, nonfragmented way. Symbolic names are given to some of the objects of the mind. These names facilitate verbal communication by pointing to mind states. It is convenient to think about mind objects as embedded in some multidimensional space, called the "mind space," spanned by axes (dimensions) corresponding to features of internal representations. The Platonic world of abstract concepts is just a small subspace of the whole mind space filled with these multidimensional mind objects. Mind space serves as an arena for all mind events.
Objects in the mind space are described by a "mind function" $M\left(X_{i}\right)$ for all relevant features $X_{i}$, playing a similar role as the probability distribution function of Nalimov (1985). Nonzero values of the mind function define these objects as fuzzy regions in the mind space. Topographical relations of objects in this space are very difficult to imagine because of the large number of dimensions involved. The mind function, defined in the mind space, represents all objects that such a system is able to recognize (i.e., correctly discriminate using partial description or distorted input). Thecognitive system is able to modify the contents of the mind space by adding more objects (learning and remembering), modifying existing objects or learning new associations (changing topographical relations between existing objects).

The creation of mind objects is elucidated by developmental psychology (Rutkowska, 1994). Mind arises from the brain, psyche from physics, during interactions forming the inner representation of the world. Symbols, or abstract labels of the mind objects, have no meaning without the mind to interpret them. They are very useful for rapid activation and structuring of the mind objects, since they are almost unique. Nonsymbolic features of mind objects are derived from sensory features and motor behavior. There is no reason why, using only symbolic names, the whole complexity of a real mind space could not be recreated. Artificial intelligence, based on the processing of symbols, does not lead to artificial minds based on the multidimensional mind objects. The meaning of the mind objects is grounded in the combination of all relevant features of their representation.

Logic and reasoning are only approximations of the dynamics of activations of objects in themind space. Expressions such as "to have in mind," "to keep in mind," "to put in mind," "to make up one's mind," and so on, refer directly to the mind space. The topography of objects in the mind space, that is, their relative distances and shared features, determine intuitive, quick responses to questions that do not leave time to think. Intuitive knowledge is identified with the quality of inner representations, formed in the process of unsupervised learning, of real objects and events in the environment in which the cognitive system devel ops.

At a given moment of time some objects may be active. The probability distribution of these active objects (corresponding to neurodynamical activity) is called the mind state. In the simplest case it is a point in the mind space, $X_{i}(t)$. Changing from one A.I. Is
change
okay? 
state of mind to another requires energy. The system receives this energy from the environment (the brain uses twenty percent of the total energy provided by metabolic processes). The external stimuli drive changes in the features of representation $X_{i}(t)$ leading to the recognition and learning processes. The internal dynamics lead to activations of the entrained mind objects (trains of thoughts or series of associations) and includes a stochastic component influencing the momentum of changes of mind states. The dynamics of the whole system is a mixture of these internal and external dynamics.

The states of mind that lead to the strongest values of the mind function leave memory traces and are remembered as "an experience," enabling feedback (reflection). This experience is evaluated in light of previous experiences ("consciously perceived") if it is active (sustained in a short-term memory) for a sufficiently long time. In the brain many other processes aretaking place, representing subconscious activity. The results of experiments on processing words and pseudowords (Pulvermueller, Preissl, Eulitz, Pantev, Lutzenberger, Elbert, \& Birbaumer, 1994) support the hypothesis that transcortical cell assemblies are involved in the recognition of mind objects. Cell assemblies are large groups of neurons, with strongly reciprocal internal connections, binding parts of the cortex in which different sensory modalities are processed. Transcortical cell assemblies are sufficient to create objects of the mind space, binding different sensory modalities in one experience, without any central place in the cortex where all information is gathered. The natural hardware realization of this function has the form of a neural network (Duch, 1996b).

There is no "mind-body problem" because from the beginning there has been no mind-body separation. Mind is a reflection of a part of the Universe in the brain/body; mind space stores all chunks of sensory and bodily experiences and the dynamics governing changes of the mind states recalls them. A picture of a bel oved person seen from a distance increases the heartbeat. Even abstract thinking can involve the body. In a vital, experiential sense, mind, being a reflection of Nature, has no boundaries and is of primary importance.

Thesymbol grounding problem (Harnad, 1990) (where does the sense of symbols come from) is solved in a straightforward way, together with the problem of qualia. An activation of a mind object is done using a subset of its features. Sinceit brings the state of mind into a specific region of the mind space, other qualities associated with this object are immediately accessible, and the back-projection paths to the sensors activated. The experience is repeated, with vividness dependent on the strength of the back-coupling and the level of activation of the object. What do I mean by "sweet"? Something sweet! The brain/mind system recreates the sensory experiences "dressed" in all associations. Discussion on grounding symbols puts the cart before the horse. Symbols are not grounded in experiences, experiences come before symbols and are labeled by symbols. The label "sweet" corresponds to a projection of all sensations, all mind objects that are associated with it. The existence of qualia has observable consequences: the probability of the next mind state obviously depends on them. "Sweet" sensation brings up memories of sweet things.

The language of the quantum mechanics of consciousness proposed by J ahn and Dunne (1987) may beuseful to describeevents in the mind space. Other problems, such as the problem of free will, also have a natural solution in the mind space model (for an in-depth discussion of the free will problem from a neuroscientific point of view, see Libet, Freeman, Sutherland, and Sutherland, 2000). The mind model sketched here allows for an explanation of many facts related to cognition (Duch, 1996a), provides a language that connects mind events to both neurophysiological events and psychological events (Duch, 1995), and is useful in creating computational models of cognitivesystems (Duch, 1996b, 1997). What has been said above should besufficient totalk about mind-body interactions in the context of anomalous experiments. Intentional states of mind activate certain parts of mind space, with objects that have active motor components. Persistent, weak activation does not enter short-term memory and thus is not experienced in a conscious way.

\section{Entanglement, Mind, and Synchronicity}

S THERE a chance that the brain is just an
instrument of the spirit? This would imply that
mental functions cannot arisein artificial systems
built on similar principles. Computational cogni-
tiveneurosciences (Gazzaniga, 1999) is a relatively
new branch of the neurosciences investigating
theoretical and computational models of neurons.
It is clear that even the simplest neural network 
models function more in a mindlike, than a computerlike, way. For example, in neural models memory has no location; it is distributed in the strength of synaptic connections between neurons. Damage to such a network leads to gradual degradation of its powers, as seen in aging people, rather than to the forgetting of specific facts. The network memory is called associative, because it can retrieve the most similar original episodes it has been trained on from fragments or associated patterns of the presented episodes. The memory has no location but is context addressableand may show errors based on phonological or semantic associations. Damaged networks may hallucinate, retrieving episodes from combination of memorized fragments. The time needed to recall a fact from a computer database is proportional to its size, since it has to be searched for facts. In neural models the time of recall is independent of the size of the database. Trying to learn too many things in a short time may lead to confusion and chaotic responses. The specific organization of the brain explains many mysterious phenomena known to psychiatrists and neuropsychologists (Parks, Levine, \& Long, 1998). Mental behavior results from brainlike information processing.

Looking at the emergent properties of neural networks, it is hard to escape the conclusion that further development al ong these lines should allow for the creation of artificial minds. This will be the ultimate test of cognitive science theories, such as the mind space theory. Theories that are more complex will be needed only when this simple, pragmatic approach fails.

This is solid science that will not go away. Why do souls and spirits becomea matter of widespread beliefs in the first place? They were the simplest solution to the problem of movement that ancient philosophers worried about. Things do not move unless they are alive, so spirits were invented to push planets and move inert bodies. Since Newton, this reason for the existence of spirits has lost its appeal. St. Thomas elaborated theA ristotelian system of the three souls: (1) the vegetative soul responsible for basic functions (roughly corresponding to metabolic processes); (2) the sensitive soul, present in animals and children, responsible for reflexes (these are sensorimotoric brain functions); and (3) the rational soul responsible for higher cognitive faculties (corresponding to frontal lobe functions). Science has explained all functions ascribed to souls and spirits, leaving these concepts empty.
Ancient (mis)conceptions were never useful in explaining anything (Lewis, 1964). In this respect they are similar tothemodern quantum approaches to consciousness (Stapp, 1993; Penrose, 1994). They lead to the identification of mind and consciousness with some kind of substance. The view presented in the previous section stresses relations between mind states. Therelational theory of mind is nonmaterialistic, although a substrate (a brain) is needed to physically realize ("materialize") mind states. Even if mind and consciousness were independent of the brain, they would have to work according to neural network principles to produce mental experiences. A "new approach" is frequently called for in view of the "crisis" in science, an approach always based on old ideas (Duch, 1994). Quantum mechanics teaches us not to claim more than we really know. Observations of synchronicity do not compel us to draw condusions of higher beings finding interest in our development, as daimed by Mansfield (1995). All that is observed are "meaningful correlations." How many strange correlations between events may happen during a lifetime?

This is very difficult to estimate, because the brain searches for meaning in whatever is experienced (Freeman, 1996). After a sleepless night or strange dreams many events may be found meaningful, especially with the human ability to reorganize memories to fit one's beliefs. A naïve estimation of such probability may be as follows. Suppose that using $\mathrm{m}$ letters (e.g., of the English al phabet) a random string of the length $\mathrm{N}$ is formed. It is then al most certain that all possible substrings of the length $\log _{m} N$ will be found in this string. Every few seconds a new perception takes place; in a year about 10 million perceptions. Assume that attention is paid to about 3,000 distinct elements forming these perceptions. Then it is almost certain that a subsequence of any two of these elements will happen. Those that have little meaning will not be remembered, but some will be evaluated as remarkable coincidences. Many factors may increase this probability to significantly higher values. It is impossible to draw inferences from incidental or anecdotal stories, although they may look very impressive. Synchronicity may only be discovered by systematic observation. If such events are fairly common (like thinking about a friend who calls at this very moment) the brain, always searching for meaning, will have to recognize some 
synchronicity events as meaningful, emotionally exciting, and worth remembering.

Synchronicity in everyday events is hard to quantify, and thus to ascribe to pure chance, or to some "acausal orderings." This quantification problem is quite serious in the remote perception experiments $(J$ ahn \& Dunne, 1987). Even though the agreement between drawings and descriptions of target events is in some cases quite striking, it is hard to express it in statistical terms. REG experiments are much closer to the reproducible experiments with synchronicity events than anything else. There are two complementary points of view here. From the parapsychological point of view they are about "micropsychokinesis, remote perception, precognitive perception," and so on ( ahn \& Dunne, 1987). F rom the synchronicity point of view there is a series of correlated events that do not seem to be causally connected. Before two sets of data are compared-human intentions and machine states, operator's reports and actual eventsnothing unusual is noticed. There may be deviations of a distribution of random events from expected statistics, but unless correlations with some acausal factors in a longer series of experiments are found, deviations may be random fluctuations. Drawings and descriptions produced during remote perception experiments become interesting only when acausal correlations with the real target events are observed.

Synchronicity correlations are sufficient to explain the anomal ous experimental data. Thequestion is: How is it possible that intentional states of mind are correlated with specific events in nature? N ontrivial correlations of entangl ed systems are possible without any interactions between them. All that is necessary are two sets of measurements. Looking at a white wall for a few minutes, the visual system, having littleinput, is working on a threshold of noise. Various mind objects areactivated in an apparently random fashion and the internal dynamics of the mind state evolution prevails. One may describe this process as a measurement: The mind (highest-level control processes) measures the activity patterns of the visual cortex. In the remote viewing experiments the mind discovers in visual patterns different objects that appear to be correlated in a nontrivial way with external events. Some results of these measurements have no correlation with later events or with target scene objects. That should be expected since only some results of the joint measurements are correlated in a nontrivial, synchronicity way. The results of these measurements are independent of the time of the second measurement (event). Nontrivial correlations may wash out for longer times due to entanglement of the brain with too many objects.

The remote perception experiences are primarily visual. The precise recognition of objects imagined or seen requires many input features. If the visual inputs are weak, inputs from the optic nerve are comparable to the natural fluctuations of activity in the visual cortex. Quantum entanglement correlates patterns of neural excitation with some other patterns slightly changing these fluctuations. This may be sufficient for recognition of elements of the picture; simple mental objects that for a brief moment are activated strongly enough to be recognized. This process takes more time than the recognition of visual scenes when inputs are strong enough to allow quick recognition of many simple objects and the retention of the picture of the whole scene as one complex object in working memory. One should experience flashes, short activations of simple objects belonging to the remote scene or event.

The dynamics of the low-level excitations in the visual system are almost chaotic in the absence of other, stronger stimuli. In the terminology of the previous paragraph, mind objects are weakly and randomly activated. This activation may be correlated, via quantum entanglement, with many objects and events in nature. Direct entanglement should be most effective (having close contact with the other person). An intention to think about a person who has visited an unknown place, or even thinking about someone who will later compare the results of the remote perception experiment with descriptions and drawings that are being made, creates experimental conditions making the mind more sensitive to objects or processes at that place. In such conditions certain mind objects are more often activated strongly enough to appear as flashes or short visions.

Experiments with Random Event Generators (REG) are also understandable from this point of view. In REG experiments, an intentional state of mind is generated and subconsciously sus- 
tained. An appropriate object of mind corresponding to the intention of obtaining positive or negative results is created in the mind space and periodically activated. Again, this activation or recognition may be described as the measurement process. On the other hand it may be interpreted as "prediction."

The entanglement mechanism proposed here allows an explanation of the strange features of these experiments:

- Effects should be small but statistically significant. Correlations between only two entangled particles involving simple photon counts are al ready hard to measure. Correlations between complex patterns involving billions of particles are measured by the brain. Most of these correlations are of a trivial nature, that is, they are acci dental or explainable by causal thought processes.

- The brain of an operator should have a cue to get entangled with the hardware equipment or with the brains of other peopleinvolved in the experiment. Avoiding direct comparison of two sets of measurements may reduce the effect. Group experiences, such as media, sport, or religious events, during which the brain is absorbed in some focused activity, should be particularly effectiveto set intentional states of the brain.

- Effects have to be intentional: Out of all possiblecorrelations with natural events one has to focus on a particular place or on a piece of equipment. Intention is necessary to start the brain process that selects associations belonging to a chain that starts with known mind objects, representing equipment or people involved in the experiment. Some people may be more skilled at, or capable of, forming such associations than others, and this should be reflected in the results.

- Consciousness does not have a direct effect on results. The role of intention is only to set the brain of the operator in a certain state. Once set, the intentional process goes on in the brain even if the operator does not pay much attention to the experiment.
- During the experiments the brain should be active in a normal, waking consciousness, but not too much distracted by external stimuli. Sensory overloading or engagement of the brain in a demanding activity may destroy the intentional process and reduce the correlations to chance level.

- Nospecial assumptions about the entangled systems are necessary. Since all systems are entangled to a similar degree, correlations should not depend on the type of noise sources in REG experiments or targets in the remote viewing experiments.

- Sincethereis no exchange of energy, only correlations, statistical results should not significantly depend on the distance between the target and the operator involved or on thetime delays between the two measurements.

- Local probabilities are never affected by quantum entanglement, only the joint probabilities of measurements are, ther efore the local data should not look "unusual." Anomalous effects should be seen only when two sets of data are compared in a series of experiments.

- The psychological effect of looking into water or gazing at a crystal, or any other activity that does not disturb the intentional states in the brain, should be favorable for remote viewing.

Most of these effects have been observed in the experiments quoted ( ahn \& Dunne, 1987). Synchronicity via quantum entangl ement seems to be the only mechanism that can explain the correlation of human intentions and mental events with the results of experiments that have been already performed (Schmidt, 1993) or that will be performed in the future. Interestingly, reexamination of a large amount of experimental data has led very recently to conclusions similar to those presented here ( $\mathrm{ahn} \&$ Dunne, 2001). In particular there seems to be no direct involvement of consciousness. It would be extremely interesting if theintentional processes in the brain could beidentified and their intensity correlated with the synchronicity effects. This 
may require identification of the cortical cell assemblies involved. At present such identification is possible only for monkeys and other animals requiring implanted el ectrodes.

It is very hard to use experimental physical techniques to discover correlations between more than a few particles. Consider a living cell, for example, a brain cell. Many biochemical processes take place every second in a cell; many of them are controlled by photon emissions and absorption providing energy for reactions. This ultraweak radiation is very coherent (Popp, 1992; Chang, Fisch, \& Popp, 1998). The pattern of these emissions and absorptions may be correlated in a very subtle way to many processes in nature, but it is not yet possible to measure such subtle effects in the laboratory. On the other hand, influences on the patterns of neural excitation in the brain should have noticeable effects on the activation of mind objects. Microtubules are good candidate structures for sensitive elements of cells that may be influenced by quantum effects (Penrose, 1994). Super-radiance and other collective quantum states should be possible in these cytoskel etal structures. Insinna (1992) has already discussed synchronicity in connection with quantum coherence in microtubules. Although quantum effects may not be useful for understanding the mind and consciousness, they may be the basis of synchronicity.

\section{Conclusions}

$\mathrm{R}$ EMOTE PERCEPTION and REG experiments seem to challenge the scientific world view (J ahn \& Dunne, 1987). A unified view of mind and nature, capable of elucidating these strange phenomena, is possible. Quantum physics provides a view of nature based on what is really known, that is, what has been measured. Cognitive science provides a view of mind as an emergent property of the brain. Occam's razor applied to this problem leads to the "minimum metaphysics" solution: Synchronicity effects arefound when two sets of measurements are compared. The proof in the Appendix shows that significant quantum entanglement effects should always be present. Other theoretical models that try to accommodate the results of anomalous experiments require either a nonlinear version of quantum mechanics, or peculiar interpretations of the quantum measurement process (Stapp, 1994).
It is very difficult to give up deeply ingrained convictions, such as those related to the separability of things, whether wethink about the objects of nature as a collection of independent bodies, or about ourselves as separated from Nature. In both cases separability, although sometimes a useful approximation, is ultimately an illusion. Many people with deep insight into their own minds and their thinking process, including Erwin Schrödinger, haveal ready written on this subject not only from the point of view of physics, but also from their own personal point of view. Once the idea of separability is given up, the results of EPR experiments, REG experiments, remoteperception, and therelation between minds and brains/bodies are understandable. Infants learn rather early to separate their body from the environment, distinguish "me" and "not-me," and the idea of separability is firmly established.

Although the calculation of quantum probabilities for such complex systems as brains is not feasible, some suggestions and the understanding of certain features of experiments involving human operators are possible. Quantum effects are not necessary to explain the cognitive mind but seem to be indispensable to an understanding of the subtle features of the mind manifested in synchronicity. A detailed theory of synchronicity should be based on the quantum mechanics of macroscopic bodies and their entanglements (Primas, 1981). There is no a priori reason why there should be no acausal correlation between brain activity and events in nature. To provethat such correlations do not exist wewould have to compute an analog of Bell's inequality for a very complex system, with wave functions for parts of the brain on one side and various natural objects on the other. Since it is impossible to separate the wave functions of any physical systems, especially macroscopic bodies, and detailed calculations of multiparticle correlations are too difficult, one has to resort to experiments. The results of experiments indicate that a small but consistent effect exists. The simplest explanation consistent with all experimental data is based on synchronicity due to quantum mechanical entanglement. The synchronicity effects described here are consistent with present theories of physics and cognitive science, and do not require any extensions of our knowledge into unknown territories. 


\section{Appendix}

$\mathrm{T}$ HIS APPENDIX provides a technical proof showing the inseparability of subsystems in quantum mechanics. State vectors representing objects belong to a Hil bert space. Operator $\mathbf{P}$ representing an observable (property, that may be observed) applied to some arbitrary state vector converts it into another state vector. Quantum mechanics is a holistic theory and does not allow for a well-defined way of describing the separation of systems. This fact gave rise to alternative formulations of mathematical foundations of quantum mechanics (Piron, 1985; Aerts, 1982), but so far all experiments show that standard quantum mechanics is correct. The Hilbert space of antisymmetric, many particle functions, describing the total system, cannot be decomposed into separate subspaces.

Consider two physical systems, $\mathrm{S}_{A}$ and $\mathrm{S}_{B}$, with $\mathrm{N}_{\mathrm{A}}$ and $\mathrm{N}_{B}$ particles (electrons or other fermions), respectively. Each system is described by its own function, $\Psi_{A}$ antisymmetric for permutations of all $\mathrm{N}_{\mathrm{A}}$ particles and $\Psi_{\mathrm{B}}$ antisymmetric for $\mathrm{N}_{\mathrm{B}}$ particles. Assuming that both functions are normalized to unity, it is easy to show that the product function $\Psi_{A B}=\Psi_{A} \Psi_{B}$ is al ways "far" from the antisymmetric function $\Psi=A \Psi_{A B}$, where $\mathbf{A}$ is the antisymmetrization operator. The distance may be measured by an overlap $\left\langle\Psi_{\mathrm{AB}} \mid \Psi\right\rangle$ or by the norm of difference: $2-\sqrt{2} \leq\left\|\Psi_{\mathrm{AB}}-\Psi\right\|^{2} \leq 2$.

The square of the norm does not exceed 2 because of the Schwartz inequal ity. The second inequality is slightly more difficult to prove. The orthonormal basis $\left\{\phi_{i}^{A}\right\}$ and $\left\{\phi_{j}^{B}\right\}$ is defined for $S_{A}$ and $S_{B}$ subsystems, and since they are separated $\left\langle\phi_{i}{ }^{A} \mid \phi_{j}{ }^{\mathrm{B}}\right\rangle=\delta_{i j}$. The unsymmetrized, normalized product functions are:

$$
\begin{aligned}
& \Phi_{A}\left(x_{1}, x_{2}, . . x_{N_{A}}\right)=\prod_{i=1}^{N_{A}} \phi_{i}^{A}\left(x_{i}\right) \\
& \Phi_{B}\left(x_{1}, x_{2}, . . x_{N_{B}}\right)=\prod_{i=1}^{N_{B}} \phi_{i}^{B}\left(x_{i}\right)
\end{aligned}
$$

The idempotent antisymmetrizer does not give proper normalization. The proper antisymmetrizer is: $\mathbf{A}_{N}=\frac{1}{\sqrt{N !}} \sum_{\mathbf{P}}(-1)^{\mathbf{P}} \mathbf{P}$, where $\mathbf{P}$ is a permutation operator and $(-1)^{\mathrm{P}}$ is its parity. Therefore $\Psi_{\mathrm{A}}$ is obtained by:

$$
\Psi_{A}\left(x_{1}, x_{2} \ldots x_{N_{A}}\right)=\mathbf{A}_{N} \Phi_{A}\left(x_{1}, x_{2} \ldots x_{N_{A}}\right)=\frac{1}{\sqrt{N !}} \sum_{\mathbf{P}}(-1)^{\mathbf{P}} \mathbf{P} \Phi_{A}\left(x_{1}, x_{2} \ldots x_{N_{A}}\right) .
$$

An analogous expression is true for $\Psi_{B}$. The antisymmetrizer $\mathbf{A}$ that creates from the product function $\Psi_{\mathrm{AB}}$ totally antisymmetric function $\Psi=\mathrm{A} \Psi_{\mathrm{AB}}$ is:

$$
\mathbf{A}=\sqrt{\frac{N_{A} ! N_{B} !}{N !}} \sum_{\mathbf{P}_{A B}}(-1)^{\mathbf{P}_{A B}} \mathbf{P}_{A B}
$$

where $\mathrm{N}=\mathrm{N}_{\mathrm{A}}+\mathrm{N}_{\mathrm{B}}$ and $\mathbf{P}_{\mathrm{AB}}$ is either identity or it permutes particles of $\mathrm{S}_{\mathrm{A}}$ with those of $\mathrm{S}_{\mathrm{B}}$. Since $\Psi$ and $\Psi_{\mathrm{AB}}$ are normalized,

$$
\begin{aligned}
& \left\|\Psi_{A B}-\Psi\right\|^{2}=\left\|\Psi_{A B}\right\|^{2}+\|\Psi\|^{2}-2\left\langle\Psi_{A B} \mid \mathbf{A} \Psi_{A B}\right\rangle= \\
& 2-2 \sqrt{\frac{N_{A} ! N_{B} !}{N !}}\left\langle\Psi_{A B} \mid \sum_{\mathbf{P}_{A B}}(-1)^{\mathbf{P}_{A B}} \mathbf{P}_{A B} \Psi_{A B}\right\rangle \geq 2-\sqrt{2} \approx 0.59
\end{aligned}
$$

because the overlap integral $\left\langle\Psi_{\mathrm{AB}} \mid \Psi\right\rangle=\left\langle\Psi_{\mathrm{AB}} \mid \mathbf{A} \Psi_{\mathrm{AB}}\right\rangle$ is nonzero only for $\mathbf{P}_{\mathrm{AB}}=$. The antisymmetric function and the product wavefunction are quite different. There is no way in quantum mechanics to go from one to the other, that is, to describe the process of separation of systems that have once interacted. 
In the textbook of A. Messiah (Messiah, 1976, Chapter XIV, §8) it is proven that this nonseparability should not matter because probabilities of different states of a spatially isolated subsystem do not depend on the antisymmetrization of the function of this subsystem with functions of all other particles in the Universe. But what about the results of joint measurements, that is correlations between observations? Consider the systems $S_{A}$ and $S_{B}$ and two independent measurements of observables corresponding to the operators $\mathbf{O}_{A}$ and $\mathbf{O}_{B}$. The wavefunctions of these systems may be expanded in the eigenbasis of:

$$
\begin{array}{ll}
\Psi_{A}=\sum_{a} C_{a}^{A} \Psi_{a}^{A} ; & \mathbf{O}_{A} \Psi_{a}^{A}=\lambda_{a} \Psi_{a}^{A} \\
\Psi_{B}=\sum_{b} C_{b}^{B} \Psi_{b}^{B} ; & \mathbf{O}_{B} \Psi_{b}^{B}=\eta_{b} \Psi_{b}^{B}
\end{array}
$$

Messiah (1976) proves that taking the total function $\Psi$ instead of the product functions $\Psi_{A} \Psi_{B}$ does not change the probabilities $\left|\mathrm{C}_{a}{ }^{\mathrm{A}}\right|^{2}$. However, he does not look at the possible correlations of joint measurements. Assuming that the two systems are separated, the result of the joint measurement is:

$$
\left\langle\Psi_{A} \Psi_{B}\left|\mathbf{O}_{A} \mathbf{O}_{B}\right| \Psi_{A} \Psi_{B}\right\rangle=\left\langle\Psi_{A}\left|\mathbf{O}_{A}\right| \Psi_{A}\right\rangle\left\langle\Psi_{B}\left|\mathbf{O}_{B}\right| \Psi_{B}\right\rangle=\left\langle\mathbf{O}_{A}\right\rangle\left\langle\mathbf{O}_{B}\right\rangle
$$

Define now a coefficient $C_{A B}$ measuring the difference between this result and the result obtained without assumption of separability, calculated with the total wave function $\Psi$ :

$$
\begin{aligned}
C_{A B} & =\left(\left\langle\Psi_{A} \Psi_{B}\left|\mathbf{O}_{A} \mathbf{O}_{B}\right| \Psi_{A} \Psi_{B}\right\rangle-\left\langle\Psi\left|\mathbf{O}_{A} \mathbf{O}_{B}\right| \Psi\right\rangle\right) /\left\langle\mathbf{O}_{A}\right\rangle\left\langle\mathbf{O}_{B}\right\rangle \\
& =1-\frac{\left\langle\Psi\left|\mathbf{O}_{A} \mathbf{O}_{B}\right| \Psi\right\rangle}{\left\langle\mathbf{O}_{A}\right\rangle\left\langle\mathbf{O}_{B}\right\rangle}
\end{aligned}
$$

If there is no difference between these two cases this coefficient should be zero. However,

$$
\begin{aligned}
\left\langle\Psi\left|\mathbf{O}_{A} \mathbf{O}_{B}\right| \Psi\right\rangle & =\frac{N_{A} ! N_{B} !}{N !} \sum_{P, Q}(-1)^{P}(-1)^{Q}\left\langle\mathbf{P}\left(\Psi_{A} \Psi_{B}\right)\left|\mathbf{O}_{A} \mathbf{O}_{B}\right| \mathbf{Q}\left(\Psi_{A} \Psi_{B}\right)\right\rangle \\
& =\frac{N_{A} ! N_{B} !}{N !}\left\langle\mathbf{O}_{A}\right\rangle\left\langle\mathbf{O}_{B}\right\rangle
\end{aligned}
$$

since all matrix elements for permutations $(\mathbf{P}, \mathbf{Q}) \neq(\mathbf{I}, \mathbf{I})$ vanish by virtue of localization of the $S_{A}$ and $\mathrm{S}_{\mathrm{B}}$ subsystems. This leads to the following inequality for the correlation coefficient:

$$
1 \geq \mathrm{C}_{\mathrm{AB}} \geq 0.5
$$

Thus there is a huge difference. Using a local description for isolated subsystems leads to correct local results, but correlations with other systems are always large, approaching perfect correlation for a large N. These results do not seem to depend on decoherence of large systems. For further discussion of separability and the role of symmetry breaking see Duch (1988). 


\section{Notes}

A preliminary version of this paper was written during theAcademy of Consciousness Meeting in Princeton, New J ersey, USA (J une// uly 1994). I am most grateful to the organizers, Robert J ahn and Brenda Dunne, for the invitation, and to the Fetzer F oundation for sponsoring this inspiring meeting.

\section{References}

Aerts, D. (1982). Description of many physical entities without the paradoxes encountered in quantum mechanics. Foundation of Physics, 12, 1131-1170.

Chalmers, D. J . (1996). The conscious mind: In search of a fundamental theory. Oxford, England: Oxford University Press.

Chang, J . J ., Fisch, J ., \& Popp, F. A. (Eds.). (1998). Biophotons. Dordrecht: Kluwer Academic.

Duch, W. (1988). Schrödinger's thoughts on perfect knowledge. In E. I. Bitsakis \& C. A. Nicolaides (Eds.), Theconcept of probability (pp. 5-14). Dordrecht: Kluwer Academic.

Duch, W. (1994). A solution to the fundamental problems of cognitive sciences. International Philosophical Preprint Exchange; this and other papers are available at: http:// www.phys.uni.torun.pl/kmk/publications.html

Duch, W. (1995). Physics of consciousness. IVth National Conference, Modeling Biological Systems (pp. 101-114), Cracow, Poland.

Duch, W. (1996a). Computational physics of the mind. Computer Physics Communications, 97, 136-153.

Duch, W. (1996b). From cognitive models to neurofuzzy systems-the mind space approach. Systems Analysis-M odeling-Simulation, 24, 53-65.

Duch, W. (1996c, December 6-7). From brain to mind to consciousness without hard problems. CognitiveSymposium, Institute of Philosophy, Poznan, Poland.

Duch, W. (1996d). Categorization, prototypetheory and neural dynamics. In T. Yamakawa \& G. Matsumoto (Eds.), Proceedings of the 4th International Conference on Soft Computing (pp. 482-485), lizuka, J apan.

Duch, W. (1997). Platonic model of mind as an approximation to neurodynamics. In S. Amari, \& N. Kasabov (Eds.), Brain-likecomputing and intel ligent information systems (pp. 491-512). Singapore: Springer.

Edelman, S. (1998). Representation is representation of similarities. Behavioral and Brain Sciences, 21, 449-498.

Einstein, A., Podolsky B., \& Rosen N. (1935). Can quantummechanical descriptions of physical reality be considered complete? Physical Review, 47, 777-780.

Freeman, W. J . (1996). Societies of brains: A study in theneuroscience of love and hate Hillsdale, NJ : Erlbaum.

Gazzaniga, M. S. (Ed.). (1999). The new cognitive neurosciences. Cambridge, MA: MIT Press.

Giulini, D., J oos, E., Kiefer, C., Kupsch, J ., Stamatescu, I. O., \& Zeh, H. D. (1996). Decoherenceand the appearance of a classical world in quantum theory. Berlin: Springer.

Gopnik, A. (1993). How we know our minds: The illusion of first-person knowledge of intentionality. Behavioral and Brain Sciences, 16, 1-14.
Harnad, S. (1990). The symbol grounding problem. Physica D, 42, 335-346.

Insinna, E. M. (1992). Synchronicity and coherent excitations in microtubules. Nanobiology, 1, 191-208.

J ahn, R. G. (1982). The persistent paradox of psychic phe nomena: An engineering perspective. Proceedings of IEEE, 70, 136-170.

J ahn, R. G., \& Dunne, B. J . (1987). Margins of reality: The role of consciousness in the physical world. New York: Harcourt Brace J ovanovich.

J ahn, R. G., \& Dunne B. J . (2001). A modular model of mind/ matter manifestations $\left(\mathrm{M}^{5}\right)$. J ournal of Scientific Exploration, 15, 299-329.

J ung, C. G., \& Pauli, W. (1973). Synchronicity. Princeton, NJ : Princeton University Press, Bollingen Series. (Originally published as Naturerklärung und Psyche Zurich: Rascher Verlag, 1952).

Kelly, G. A. (1955). Thepsychol ogy of personal constructs. New York: Norton.

Khalfin, L. A., \& Tsirelson, B. S. (1992). Quantum/classical correspondence in the light of Bell's inequalities. Foundation of Physics, 22, 879-948.

Laurikainen, K. V. (1988). Beyond theatom. Berlin: Springer.

Leopold, D. A., \& Logothetis, N. K. (1999). Multistable phe nomena: Changing views in perception. Trends in Cognitive Studies, 3(7), 254-265.

Lewin, K. (1938). The conceptual representation and themeasurement of psychol ogical forces. Durham, NC: DukeUniversity Press.

Lewis, C. S. (1964). The discarded image. Cambridge, England: Cambridge University Press.

Libet, B., Freeman, A., Sutherland, K., \& Sutherland, J . K. B. (Eds.). (2000). The volitional brain: Towards a neuroscience of free will. Exeter, England: I mprint Academic.

Mansfield, V. (1995). Synchronicity, scienceand soul-making. Chicago: Open Court.

Mehra, J ., \& Wightman, A. S. (Eds.). (1995). The collected works of Eugene Paul Wigner (Vol. VI). Berlin: Springer.

Messiah, A. (1976). Quantum mechanics, Vol . II . Amsterdam: North Holland.

Nalimov, V. V. (1985). Space, time and life: The probabilistic pathways of evolution. Philadel phia: ISI Press.

Nielsen, M. A., \& Chuang I. L. (2000). Quantum computation and quantum information. Cambridge, England: Cambridge University Press.

O'Regan, J . K., \& Noë, A. (2001, in press). A sensorimotor account of vision and visual consciousness. Behavioral and brain sciences, 24(5).

Parks, R. W, Levine, D. S., \& Long, D. (E ds.). (1998). Fundamentals of neural network modeling. Cambridge, MA: MIT Press.

Pauli, W., \& J ung, C. G. (1992). In C.A. Meier (Ed.), Ein Briefweksel, 1932-1958. Berlin: Springer. (Published in English as Atom and archetype: The Pauli/ J ung letters, 1932-1958, C. A. Meier, Ed., \& D. Roscoe, Trans. Princeton, NJ : Princeton U niversity Press, 2001)

Penrose, R. (1994). Shadows of the mind. Oxford, England: Oxford University Press.

Piron, C. (1985). Quantum mechanics: Fifty years later. In P. Lahti \& P. Mittel staedt (Eds.), Symposium on the foundations of modern physics (pp. 207-216). Singapore: World Scientific. 
Popp, F. (E d.), (1992). Recent advances in bi ophoton research and its applications. Singapore: World Scientific.

Primas, H. (1981). Chemistry, quantum mechanics and re ductionism, Lecture Notes in Chemistry 24. Berlin: Springer.

Pulvermueller, F., Preissl, H., Eulitz, C., Pantev, C., Lutzenberger, W., Elbert, T., \& Birbaumer, N. (1994). Psycol oquy, 5(48), brain-rhythms.1.pulvermueller.

Putnam, H. (1978). Many faces of realism. La Salle, IL: Open Court.

Puthoff, H., \& Targ C. (1976). A perceptual channel for information transfer over kilometer distances: Historical perspective and recent research. Proceedings of IEEE, 64, 329354.

Rakover, S. S. (1993). Precis of metapsychol ogy: Missing links in behavior, mind, and science. Psycoloquy, 4(55), metapsychology. 1.rakover.

Ruppin, E. (1995). Neural modeling of psychiatric disorders. Network, 6, 636-656.

Rutkowska, J . (1994). The computational infant. Oxford, England: Oxford University Press.

Schmidt, H. (1993). Observation of a psychokinetic effect under highly controlled conditions. J ournal of Parapsychology, 57, 351-372.

Selleri, F. (1987). History of the EPR paradox, In F. Selleri (Ed.), Quantum mechanics versus local realism: The Einstein-Podolsky-Rosen paradox (pp. 1-61). New York: Plenum Press.

Shaw, M. L. G., \& Gaines B. R. (1992). Kelly's “Geometry of psychological space" and its significance for cognitivemodeling. New Psychologist, 10, 23-31.

Shepard, R. N. (1987). Toward a universal law of generalization for psychological science. Science, 237, 1317-1323.

Shepard, R. N. (1993). On the physical basis, linguistic representation, and conscious experience of colors. In G. Harman (Ed.), Conceptions of the mind (pp. 217-245). Hillsdale, NJ : Erlbaum.

Shepard, R. N. (1994). Perceptual-cognitive universals as reflections of the world. Psychonomic Bulletin \& Review, 1, 2-28.

Stapp, H. P. (1993). Mind, matter and quantum mechanics. Berlin: Springer.

Stapp, H. P. (1994). Theoretical model of a purported empirical violation of the predictions of quantum theory. Physical Review A, 50, 18-22.

Tegmark, M. (2000). Theimportance of quantum decoherence in brain processes. Physical Review E, 61, 4194-4206.

Wang, P. P. (Ed.), (2001). Computing with words. New York: Wiley.

Wigner, E. P. (1962). In I. J . Good (Ed.), The scientist speculates: An anthology of partly-baked ideas (pp. 284-302). London: Heinemann.

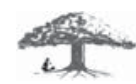

